\title{
First isolation of dengue virus from Lao PDR in a Chinese traveler
}

\author{
Xiaofang Guo ${ }^{1,2}$, Qiumin Zhao ${ }^{1}$, Chao Wu², Shuqing Zuo ${ }^{1}$, Xiaoai Zhang ${ }^{1}$, Na Jia ${ }^{1}$, Jiangyun Liu³ \\ Hongning Zhou ${ }^{2^{*}}$ and Jiusong Zhang ${ }^{*}$
}

\begin{abstract}
Background: Epidemic dengue activity has been demonstrated in several southern regions of China, but not in Yunnan province, which borders countries in Southeast Asia where dengue is endemic. Many dengue cases imported from Southeast Asia to Yunnan have been reported, but dengue virus (DENV) has not been isolated from any patients. This study is the first to report the isolation of DENV from a Chinese traveler returning to Yunnan from LaO PDR.
\end{abstract}

Findings: A serum sample was collected from a patient presenting with a febrile illness who returned from Lao PDR in 2009 and was used to inoculate Aedes albopictus C6/36 cells for viral isolation. The viral isolate was identified using reverse transcription-polymerase chain reaction, and phylogenetic analyses based on the full $E$ sequence were performed using Clustalx 1.8 software. The analyses detected DENV genome, and thus, a DENV isolate was obtained from the patient's serum sample. The new DENV isolate was grouped into genotype Asia 1, serotype 2. The viral E protein shared the greatest nucleotide sequence identity (99.6\%) with the D2/Thailand/0606aTw strain isolated from Thailand in 2006 and demonstrated $94.3 \%$ to 100\% identity with the predicted amino acid sequence of other DENV 2 strains.

Conclusions: Our findings indicate that DENV serotype 2 is circulating in Lao PDR, and surveillance of patients suspected of infection with dengue should be conducted not only by a serological test but also by pathogenic detection methods.

Keywords: Dengue virus, Isolation, Envelope protein, Phylogenetic analysis

\section{Introduction}

Dengue virus (DENV), a member of the Flavivirus genus of the Flaviviridae family, has four distinct serotypes (DENV 1 to 4 ) that cause a spectrum of disease ranging from asymptomatic, mild, undifferentiated fever and classical dengue fever to the severe disease known as dengue hemorrhagic fever/dengue shock syndrome [1]. Dengue has emerged as a major mosquito-borne viral disease in tropical and subtropical regions over the past 20 years. The disease is prevalent in Africa, the Americas, the Eastern Mediterranean, Southeast Asia and the Western Pacific. An estimated 2.5 billion people live in areas where dengue is endemic, and the annual incidence of the disease

\footnotetext{
* Correspondence: zhhn@yipd.org; zhang_jiusong@yahoo.com.cn

${ }^{2}$ Yunnan Institute of Parasitic Diseases, Pu'er, Yunnan, People's Republic of China

${ }^{1}$ State Key Laboratory of Pathogen and Biosecurity, Beijing Institute of

Microbiology and Epidemiology, Beijing, People's Republic of China

Full list of author information is available at the end of the article
}

in these areas is approximately 50 million cases. In the last 50 years, the incidence of the disease has increased $>30$ fold due to increasing geographic expansion and, in the present decade, a move from urban to rural areas $[2,3]$.

In China, epidemic dengue activity has been detected in the southern regions, primarily the provinces of Guangxi, Guangdong, Fujian, Zhejiang and Hainan [4]. From 1978 to 2008, a number of dengue outbreaks were reported, comprising a total of 655,324 cases and resulting in 610 deaths [5]. Yunnan Province, located in southern China, has a tropical to subtropical climate and borders Vietnam, Lao PDR and Myanmar, countries in which dengue is endemic [6-8]. Although no dengue outbreak has been recorded, imported cases have frequently been reported in Yunnan. In 2008, 77 imported cases and 12 indigenous cases (on the border with Myanmar) were reported, all of which were diagnosed by a serological test [9].

\section{Biomed Central}


In the present study, we report the isolation of a DENV 2 strain from a patient with a febrile illness who returned from Lao PDR in 2009. This is the first report of DENV isolated from an individual who had traveled to Lao PDR.

\section{Materials and methods}

This study was performed after consultation with the patient and receipt of written consent. All study-related information was acquired and used anonymously. The Institutional Review Board of the Beijing Institute of Microbiology and Epidemiology approved the research involving human subjects.

A 22-year-old man with symptoms of persistent fever $\left(>39^{\circ} \mathrm{C}\right)$ and headache visited a local health center in China at the China-Lao PDR border on October $16^{\text {th }}$, 2009. The patient had been living in Muong Xay Town, Lao PDR, which is located in the north of Lao PDR, for six months as a part-time worker before his visit to the health center (Figure 1). Muong Xay Town and the China-Lao PDR border are approximately 100 kilometers apart. The onset of illness occurred on October $15^{\text {th }}$,
2009. Because there was no effective treatment available in Muong Say Town, the patient returned to Yunnan to visit a Chinese doctor one day after his symptoms began. In accordance with a routine surveillance program, a serum sample was collected from the patient on the day he visited the doctor, and it was tested for immunoglobulin (Ig) G and IgM antibodies to DENV using a rapid test card (OneStep Dengue RapiCard ${ }^{\mathrm{TM}}$ InstaTest, Cortez Diagnostics Inc., Calabasas, California, USA). This test is the standard method used for the rapid diagnosis of DENV infections. The test was negative. No skin rashes or other systemic symptoms were apparent. The patient was hospitalized as a suspected case of dengue fever for three days until defervescence.

DENV genome in serum was detected by reverse transcription-polymerase chain reaction (RT-PCR). Briefly, total RNA was extracted from the patient's serum sample using Trizol and was reverse-transcribed using random hexamers to obtain the first-strand cDNA. The PCR was performed with a pair of DENV universal primers, which amplified the partial capsid-premembrane genes (511 bp).

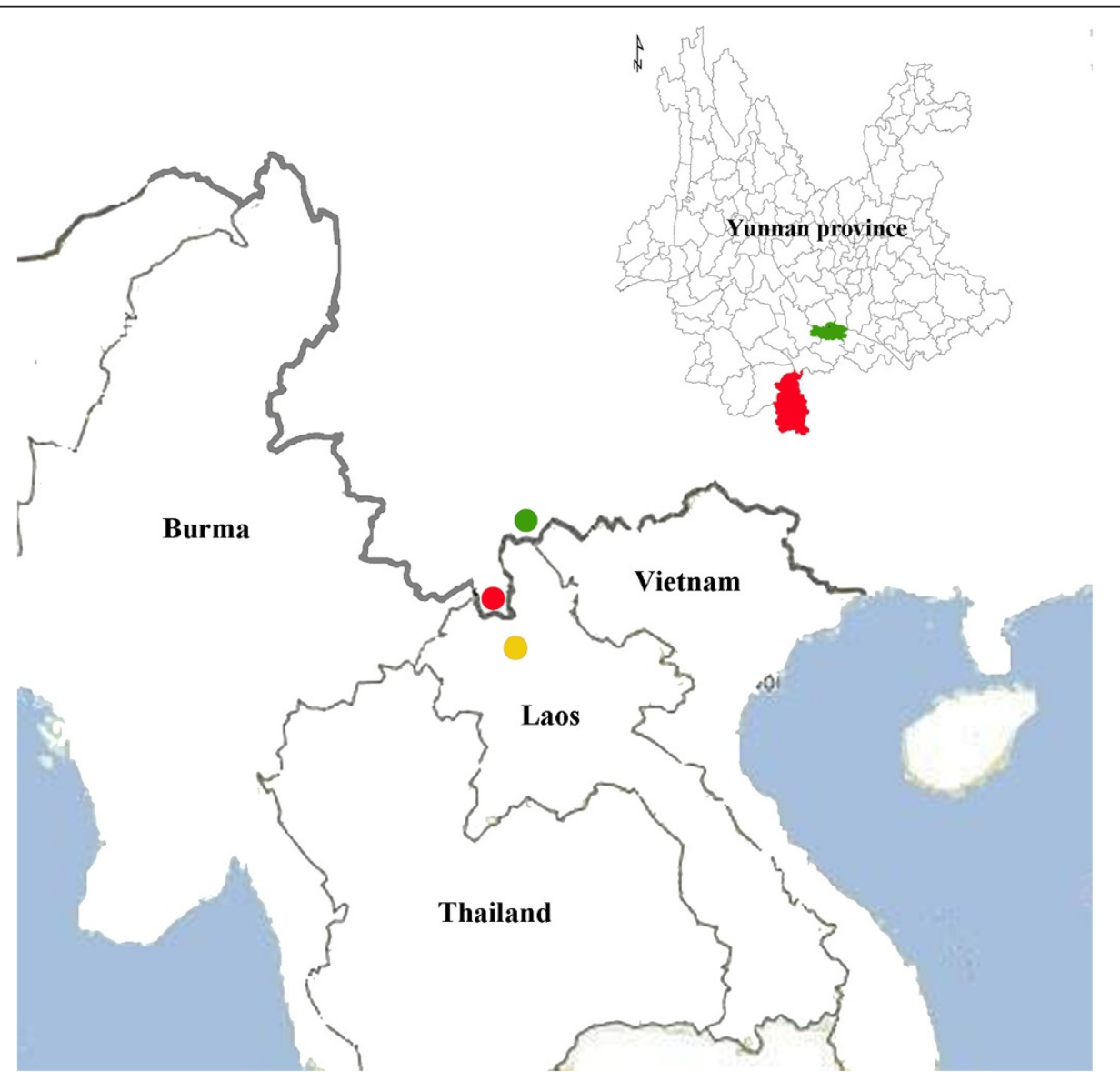

Figure 1 Map of the Yunnan province and surrounding regions. Yellow dot indicates location of DENV-infected site. Red dot and shading indicate location of diagnosed site. Green dot and shading indicate location of living site of the patient. 

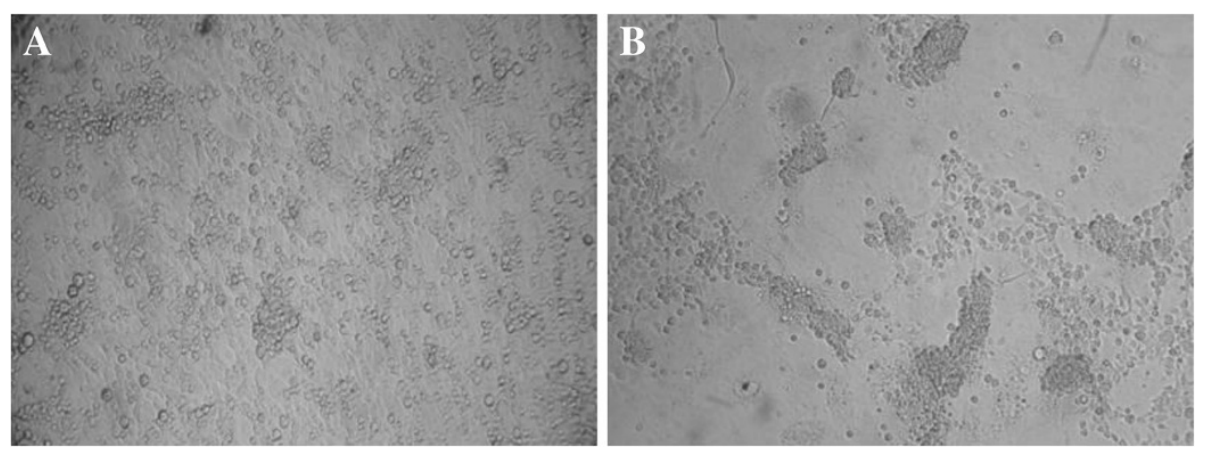

Figure 2 Viral isolate MLDENV-09-induced cytopathic effect (CPE) on C6/36 cells. A: normal C6/36 cells; B: virus-infected C6/36 cells.

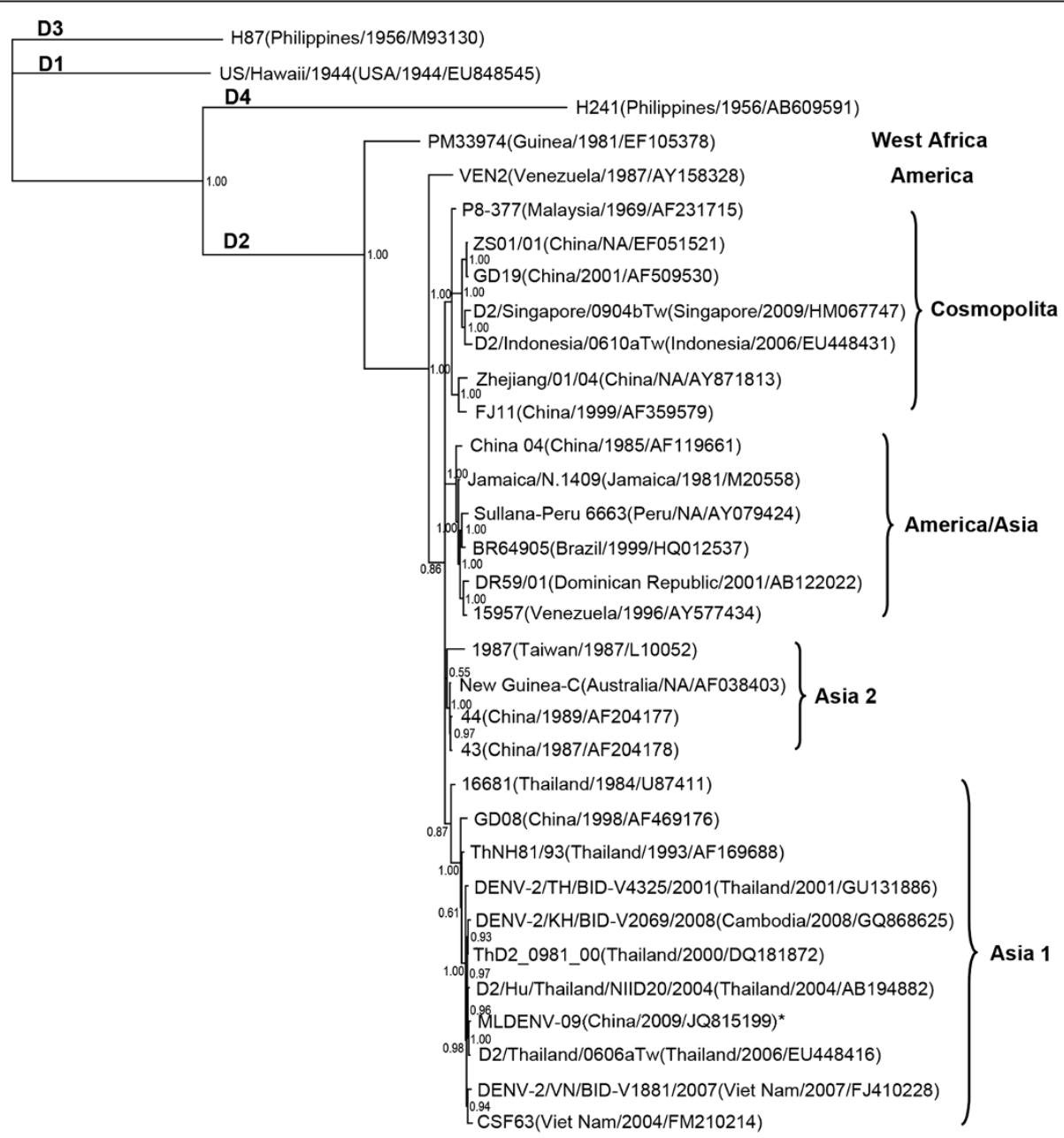

Figure 3 Phylogenetic tree based on the full E gene of the reference DENV strains. DENV 3 was used as out-groups. The strains are denoted by the strain name, country of isolation, year of isolation and GenBank accession number. "NA" means "not available." DENV serotypes 1-4 are shown as D1-D4, respectively. An asterisk is used to denote the new isolate identified in this study. 
A semi-nested PCR was then performed using the typespecific primers described by Lanciotti [10]. The PCR products were directly sequenced using an automated DNA sequencer (ABI PRISM 373, Perkin-Elmer, USA). The DNA was sequenced using both forward and reverse primers to verify the sequences. The sequences were then subjected to a BLAST search.

The serum specimen that was positive for DENV was diluted 1:10 in RPMI-1640 medium containing 2\% fetal calf serum and cultured with Aedes albopictus C6/36 cells at $32^{\circ} \mathrm{C}$ for viral isolation. The cultures were examined daily for evidence of a virus-induced cytopathic effect (CPE). The culture supernatant was filtered with a $0.22-\mu \mathrm{m}$ filter and then blind-passaged. The isolate was then identified using a DENV-specific RT-PCR as described above.

A pair of primers (Den2-EF: AAC ATG GAT GTC ATC AGA AGG; Den2-ER: CCA ATC TTG TTA CTG AGC GG) was used to amplify the envelope (E) gene of the virus from a cell culture infected with the isolate. The $\mathrm{E}$ sequence generated in this study was aligned with 32 reference sequences downloaded from GenBank, including all four serotypes of DENV. A pairwise analysis between the strains was performed using the DNAstar program. Multiple sequence alignments were carried out using Clustalx 1.8 software [11]. Phylogenetic trees were generated using the Bayesian Metropolis-Hastings Markov Chain Monte Carlo (MCMC) treesampling methods implemented by Mr. Bayes 3.1 software. We used the GTR model as the evolutionary model with gamma distributed rate variation across sites and a proportion of invariable sites [12]. The run was stopped when the standard deviation of split frequencies was below 0.01 . A DENV 3 strain H87 sequence was used as an out-group control.

\section{Results and discussion}

The patient's serum was positive for DENV genome as determined by RT-PCR using DENV universal primers. After amplification with type-specific primers, a 119-bp nucleotide segment was obtained, which matched serotype 2 of DENV. When the serum specimen was used to inoculate C6/36 cells, a significant CPE was observed in the second passage on day 6 after inoculation. Cell fusion characterized the CPE (Figure 2). The viral isolate, designated as MLDENV-09, was positive for DENV RNA by RT-PCR with the universal primers. The isolate was identified as DENV serotype 2 by semi-nested PCR using the typespecific primers and a BLAST search. Furthermore, the full $\mathrm{E}$ sequence of $1485 \mathrm{bp}$ was obtained and deposited into the GenBank database under the accession number JQ815199.

The phylogenetic tree based on the full $E$ sequences of 30 DENV 2 and the reference DENV 1, 3 and 4 strains is shown in the Figure 3. Separately from the US/Hawaii/ 1944, H87 and H241 strains, which are recognized as DENV serotypes 1, 3 and 4, respectively, the 30 sequences of DENV 2 were grouped into three distinct phylogenetic groups with high bootstrap support. The PM33974 and VEN2 strains, which belonged to West Africa and America genotypes, respectively, were classified into their own clade. Another cluster consisted of 28 closely related sequences that branched into four sub-clades generally recognized as the Asia 1, Asia 2, America/Asia and Cosmopolitan genotypes. The newly isolated MLDENV09 strain was grouped into the Asia 1 clade with 10 other strains isolated from 1984 to 2008, including 9 from Southeast Asia (Thailand, Vietnam and Cambodia) and one Chinese sequence, GD08/98.

When compared with the other Asia 1 strains used in this study, the new isolate, MLDENV-09, had 95.6\%99.6\% nucleotide sequence identity and 97\%-100\% amino acid sequence identity. The MLDENV-09 strain had the greatest nucleotide sequence similarity (99.6\%) with the D2/Thailand/0606aTw strain isolated from Thailand in 2006, and it had 100\% amino acid sequence identity with two Thai strains, ThD2_0981_00 and D2/ $\mathrm{Hu} /$ Thailand/NIID20/2004.

Many cases of dengue with pathogenic presentation have been imported from Southeast Asia to China, but the introduction of dengue from Lao PDR has never been reported prior to the case described here [13,14]. To our knowledge, this is the first isolation of DENV from Lao PDR. The phylogenetic analysis based on the viral E gene, which is generally used to identify the DENV genotype [15], showed that the new isolate belonged to serotype 2 of DENV and was classified into the Asia 1 genotype (Figure 3).

No dengue endemic has been detected in Yunnan to date. However, there is a greater potential for a dengue epidemic in Yunnan under the current circumstances, as people and materials frequently transfer during frontier trade and tourism on the border of Yunnan. Moreover, one of the competent vectors for DENV, the Aedes albopictus mosquito, is distributed throughout the Yunnan province [16]. Therefore, if DENV invades, an outbreak could occur in Yunnan. Through surveillance mechanisms, we detected an imported dengue case in a person returning from Lao PDR who was experiencing the acute-phase of infection with DENV, when specific antibodies were not yet apparent, but a viral isolate was obtained.

In conclusion, our findings indicate that laboratorybased surveillance for dengue is of great importance to prevent disease outbreaks. Sentinel clinics located in high-risk areas should reinforce surveillance of febrile cases using serological detection, viral nucleotide screening and virus isolation. 


\section{Abbreviations}

DENV: Dengue virus; RT-PCR: Reverse Transcription-Polymerase chain reaction; CPE: Cytopathic Effect; E: Envelope.

\section{Competing interests}

The authors declare that they have no competing interests.

\section{Authors' contributions}

$\mathrm{JZ}$ and $\mathrm{HZ}$ conceived and designed the experiments. XG and QZ performed the experiments. SZ, XZ and NJ analyzed the data. CW performed the serological test. $J \mathrm{~L}$ collected the information and serum sample of the patient. XG and JZ wrote the paper. $\mathrm{HZ}$ was responsible to contact with the local health center where the patient visited. All authors read and approved the final manuscript.

\section{Acknowledgments}

This research was supported by the National Natural Science Fund of China (No.81273138, 30960327, and 30660160).

\section{Author details}

${ }^{1}$ State Key Laboratory of Pathogen and Biosecurity, Beijing Institute of Microbiology and Epidemiology, Beijing, People's Republic of China. ${ }^{2}$ Yunnan Institute of Parasitic Diseases, Pu'er, Yunnan, People's Republic of China. ${ }^{3}$ Mengla County Center for Diseases Control and Prevention, Xishuangbanna, People's Republic of China.

Received: 15 May 2012 Accepted: 12 February 2013

Published: 4 March 2013

\section{References}

1. WHO/World Health Organization: Dengue haemorrhagic fever: diagnosis, treatment and control. Geneva, Switzerland: WHO; 1997.

2. Gubler DJ: Epidemic dengue/dengue hemorrhagic fever as a public health, social and economic problem in the 21 st century. Trends Microbiol 2002, 10:100-103.

3. Gubler DJ: The changing epidemiology of yellow fever and dengue, 1900-2003: full circle? Comp Immunol Microbiol Infect Dis 2004 27:319-330.

4. Gao XY, Nasci R, Liang GD: The neglected arboviral infections in mainland China. PloS Negl Trop Dis 2010, 4:e624.

5. Wu JY, Lun ZR, James AA, Chen XG: Dengue fever in mainland China. AmJTrop Med Hyg 2010, 83:664-671.

6. Rabaa MA, Ty Hang VT, Wills B, Farrar J, Simmons CP, Holmes EC: Phylogeography of recently emerged DENV-2 in Southern Vietnam. PLoS Negl TropDis 2010, 4:e766.

7. Fukunaga T, Phommasack B, Bounlu K, Saito M, Tadano M, Yoshihiro M, Kazumi K, Sakae A, Masaki S: Epidemiological situation of dengue infection in Lao P.D.R. Trop Med 1993, 35:219-227.

8. Thu HM, Lowry K, Myint TT, Shwe TN, Han AM, Khin KK, Thant KZ, Thein S, Aaskov J: Myanmar dengue outbreak associated with displacement of serotypes 2, 3, and 4 by dengue 1. Emerg Infect Dis 2004, 10:593-597.

9. Guo XF, Wu C, Wang PY, Mao XH, Zhou HN, Yang J, Dong SH, Ling YX, Gao SX: Serological investigation of dengue in Western Yunnan. Chin J Zoonoses 2010, 26:502-507. In Chinese.

10. Lanciotti RS, Calisher CH, Gubler DJ, Chang GJ, Vorndam AV: Rapid detection and typing of dengue viruses from clinical samples by using reverse transcriptase-polymerase chain reaction. J Clin Microbiol 1992, 30:545-551

11. Thompson JD, Higgins DG, Gibson TJ: CLUSTAL W: improving the sensitivity of progressive multiple sequence alignment through sequence weighting, position-specific gap penalties and weight matrix choice. Nucleic Acids Res 1994, 22:4673-4680.

12. Ronquist F, Huelsenbeck JP: MRBAYES 3: Bayesian phylogenetic inference under mixed models. Bioinformatics 2003, 19:1572-1574.

13. Xia SC, Yan JY, Lu YY, Weng JQ, Mao HY, Xu CP: Identification of dengue virus type 2 isolated from Zhejiang Province and its molecular epidemiology. Chin J Vector Bio Control 2006, 17:404-408. In Chinese.

14. Xu GZ, Dong HJ, Shi NF, Liu SJ, Zhou AM, Cheng ZH, Chen GH, Liu JY, Fang T, Zhang HW, Gu CY, Tan XJ, Ye JJ, Xie SY, Cao GW: An outbreak of dengue virus serotype 1 infection in Cixi, Ningbo, People's Republic of China,
2004, associated with a traveler from Thailand and high density of Aedes albopictus. AmJTrop Med Hyg 2007, 76:1182-1188.

15. Rico-Hesse R: Microevolution and virulence of dengue viruses. Adv Virus Res 2003, 59:315-341.

16. Dong XS, Zhou HN, Gong ZD: The mosquito fauna of Yunnan, China, Volume 2. Yunnan: Yunan Science \&Technology Press; 2010:82. In Chinese.

doi:10.1186/1743-422X-10-70

Cite this article as: Guo et al:: First isolation of dengue virus from Lao PDR in a Chinese traveler. Virology Journal 2013 10:70.

\section{Submit your next manuscript to BioMed Central and take full advantage of:}

- Convenient online submission

- Thorough peer review

- No space constraints or color figure charges

- Immediate publication on acceptance

- Inclusion in PubMed, CAS, Scopus and Google Scholar

- Research which is freely available for redistribution

Submit your manuscript at www.biomedcentral.com/submit 